\title{
CERTAIN DETERMINANTS RELATED TO THE VANDERMONDE
}

\author{
A. M. FINK
}

\begin{abstract}
We find a formula for determinants similar to the Vandermonde. These determinants arise from a problem in differential equations.
\end{abstract}

In attempting to find inequalities of the form $\int_{0}^{1} f^{2} \leqq k^{2} \int_{0}^{1}\left(f^{(n)}\right)^{2}$ when $f$ has $n$ zeros in $(0,1)$, one finds that the extremals for this problem, say $f$, satisfy differential equations of the form $f^{(2 n)}=(-1)^{n} \lambda^{n} f$ with boundary conditions of the form $f^{(k)}(0)=0$ for $k=0, \cdots, \alpha-1, n, \cdots, n+\beta-1$ and $f^{(k)}(1)=0$ for $k=0, \cdots, \beta-1, n, \cdots, n+\alpha-1$ where $\alpha+\beta=n$. Finding $\lambda$ for which this problem has nontrivial solutions is equivalent to finding the zeros of a large determinant whose typical column is

$$
\begin{array}{r}
\left(1, \omega, \omega^{2}, \cdots, \omega^{\alpha-1}, \omega^{n}, \omega^{n+1}, \cdots, \omega^{2 n-\alpha-1}, e^{\omega \lambda}, \omega e^{\omega \lambda}, \cdots,\right. \\
\left.\omega^{\alpha-1} e^{\omega \lambda}, \omega^{n} e^{\omega \lambda}, \cdots, \omega^{2 n-\alpha-1} e^{\omega \lambda}\right)^{T} .
\end{array}
$$

Here $\omega$ is a $2 n$th root of $(-1)^{n}$, one column for each root. Using the Laplace expansion by minors of the first $n$ rows, one is led to consider determinants of the form $V(\alpha, n, m)$. The ith column of $V(\alpha, n, m)$ is $\left(1, x_{i}, x_{i}^{2}, \cdots, x_{i}^{\alpha}, x_{i}^{m}, \cdots, x_{i}^{m+n-\alpha-2}\right)^{T}$, for $\alpha \geqq 0, n \geqq \alpha+2, \quad m \geqq \alpha+1$, and all integral. If $m=\alpha+1$ then $V(\alpha, n, m)$ is the familiar Vandermonde determinant which we denote by $V_{n}\left(x_{1}, \cdots, x_{n}\right)$. Finding a formula for $V(n-2, n, m)$ is equivalent to a problem posed by Hirschhorn [1].

By an argument entirely analogous to one applied to the Vandermonde, it is easy to see that $x_{i}-x_{j}, i \neq j$, is a factor of $V(\alpha, n, m)$ and hence so is $V_{n}\left(x_{1}, \cdots, x_{n}\right)=\prod_{j>i}\left(x_{j}-x_{i}\right)$. Consequently we write

$$
V(\alpha, n, m)\left(x_{1}, \cdots, x_{n}\right)=V_{n}\left(x_{1}, \cdots, x_{n}\right) g(\alpha, n, m)\left(x_{1}, \cdots, x_{n}\right),
$$

and attempt to compute the function $g(\alpha, n, m)$.

We can note some elementary properties of $g(\alpha, n, m)$. A permutation of the variables in $V(\alpha, n, m)$ being a permutation of the columns may change the sign, however, it is the same change as that produced in $V_{n}$, thus $g(\alpha, n, m)$ remains unchanged. So $g(\alpha, n, m)$ is a symmetric function

Received by the editors August 29, 1972.

AMS (MOS) subject classifications (1970). Primary 15A15.

Key words and phrases. Vandermonde, extremal. 
of $n$ variables. Next, each term in $V(\alpha, n, m)$ has joint degree $1+\cdots+$ $\alpha+m+\cdots+(m+n-\alpha-2)$ while each term in $V_{n}$ has joint degree $1+2+\cdots+(n-1)$ so that each term in $g(\alpha, n, m)$ has joint degree $(n-\alpha-1)(m-\alpha-1)$.

The plan of the computation is to prove the correct formula for $g(n-2, n, m)$ by induction on the number of variables. This is exactly the problem posed by Hirschhorn [1]. Then this identity is used to complete the induction for the rest of the functions.

To simplify the notation we write $x$ for the vector $\left(x_{1}, \cdots, x_{n}\right)$, the size to be understood from the context, and write $x_{i}^{\prime}$ for the vector $\left(x_{1}, \cdots, x_{i-1}, x_{i+1}, \cdots, x_{n}\right)$, that is $x_{i}$ is deleted. For the product $\left[\prod_{j \neq i}\left(x_{i}-x_{j}\right)\right]^{-1}$ we simply write $\pi_{i}$. We note finally that

$$
V_{n}(x)^{-1} V_{n-1}\left(x_{i}^{\prime}\right)=(-1)^{n-i} \pi_{i} .
$$

We now look at $V(\alpha, n, m)$ and expand by elements of the last row. We get

$$
V(\alpha, n, m)(x)=\sum_{i=1}^{n}(-1)^{n+i} x_{i}^{m+n-\alpha-2} V(\alpha, n-1, m)\left(x_{i}^{\prime}\right) .
$$

First we take $\alpha=n-2$ and note that $V(n-2, n-1, m)$ has not been defined, but in this case the correct replacement for the symbol $V(n-2, n-1, m)\left(x_{i}^{\prime}\right)$ is $V_{n-1}\left(x_{i}^{\prime}\right)$, i.e. we may define $g(n-1, n, m) \equiv 1$. Now we divide both sides of identity (1) by $V_{n}(x)$ to derive the basic recursion for the function $g(\alpha, n, m)$,

$$
g(\alpha, n, m)(x)=\sum_{i=1}^{n} x_{i}^{m+n-\alpha-2} \pi_{i} g(\alpha, n-1, m)\left(x_{i}^{\prime}\right) .
$$

Specializing to $\alpha=n-2$ we get

$$
g(n-2, n, m)(x)=\sum_{i=1}^{n} x_{i}^{m} \pi_{i}
$$

Finally, we look at identity (1) when $0 \leqq m=\alpha \leqq n-2$. Then $V(n-2, n, m)$ has two identical rows so is zero and $V(\alpha, n-1, m)\left(x_{i}^{\prime}\right)$ is to be replaced by $V_{n-1}\left(x_{i}^{\prime}\right)$. Again dividing by $V_{n}(x)$ we get the useful identity

$$
\sum_{i=1}^{n} x_{i}^{m} \pi_{i}=0 \text { for } n \geqq 2, m=0,1, \cdots, n-2 .
$$

The form of the formula for $g(n-2, n, m)$ is not satisfactory since it involves a division. We improve the representation in Lemma 1 , whose conclusion is the problem mentioned above. 
LEMMA 1. For $m>n-2$,

$$
\sum_{i=1}^{n} x_{i}^{m} \pi_{i}=\sum_{|k|=m-n+1} x_{1}^{k_{1}} x_{2}^{k_{2}} \cdots x_{n}^{k_{n}},
$$

where the sum on the right is to be interpreted as the sum over all vectors $\left(k_{1}, \cdots, k_{n}\right)$ with $k_{i} \geqq 0, k_{i}$ integral, and $|k|=k_{1}+\cdots+k_{n}=m-n+1$.

Proof. First, if $m=n-1$, then the left-hand side of (5) is

$$
\begin{aligned}
g(n-2, n, n-1)(x) & =V(n-2, n, n-1)(x) V_{n}(x)^{-1} \\
& =1=\sum_{|k|=0} x_{1}^{k_{1}} x_{2}^{k_{2}} \cdots x_{n}^{k_{n}} .
\end{aligned}
$$

Thus let $m \geqq n$ and we do an induction on the number of variables. The cases $n=1$ and 2 are easily verified, $\pi_{i} \equiv 1$ when there is only one variable. Then

$$
\begin{aligned}
\sum_{|k|=m-n+1} x_{1}^{k_{1}} \cdots x_{n}^{k_{n}} & =\sum_{k_{1}=0}^{m-n+1} x_{1}^{k_{1}}\left(\sum_{|k|=m-n+1-k_{1}} x_{2}^{k_{2}} \cdots x_{n}^{k_{n}}\right) \\
& =\sum_{k_{1}=0}^{m-n+1} x_{1}^{k_{1}} \sum_{i=2}^{n} x_{i}^{m-k_{1}-1}\left[\prod_{j \neq i ; j \neq 1}\left(x_{i}-x_{j}\right)\right]^{-1} \\
& =\sum_{k_{1}=0}^{m-n+1} \sum_{i=2}^{n} x_{1}^{k_{1}} x_{i}^{m-k_{1}-1} \pi_{i}\left(x_{i}-x_{1}\right)
\end{aligned}
$$

where we have used the induction hypothesis. The last outer sum now telescopes and becomes

$$
\sum_{i=2}^{n} x_{i}^{m} \pi_{i}-x_{1}^{m-n+2} \sum_{i=2}^{n} x_{i}^{n-2} \pi_{i},
$$

and the proof is completed if we can show that $-x_{1}^{m-n+2} \sum_{i=2}^{n} x_{i}^{n-2} \pi_{i}=$ $x_{1}^{m} \pi_{1}$. But this is equivalent to (4).

We now introduce the symbol $\sigma(j, n)$ to be defined by

$$
\begin{aligned}
\sigma(j, n) & =0 \quad \text { if } j<0 \\
& =1 \text { if } j=0 \\
& =\sum_{|k|=j} x_{1}^{k_{1}} x_{2}^{k_{2}} \cdots x_{x_{n}}^{k_{n}} .
\end{aligned}
$$

We can then combine (3), (4) and (5) into one identity.

$$
g(n-2, n, m)=\sigma(m-n+1, n), \quad n \geqq 2 .
$$

We intend to use the recursion (2) where (7) gives the right-hand side in the first step of an induction. We need an identity that we label Lemma 2. 


\section{LEMMA 2.}

$$
\sigma(j, n-1)=\sigma(j, n)-x_{i} \sigma(j-1, n)
$$

where the left-hand side is evaluated at $x_{i}^{\prime}$ and the right-hand side at $x$.

Proof. The cases when $j \leqq 1$ are easily verified and when $j>1$ we merely write $\sigma$ as a polynomial in $x_{i}$. Then

$$
\begin{aligned}
& \sigma(j, n)(x)=\sum_{k=0}^{j} x_{i}^{j-k} \sigma(k, n-1)\left(x_{i}^{\prime}\right) \text { and } \\
& \sigma(j-1, n)(x)=\sum_{k=0}^{j-1} x_{i}^{j-1-k} \sigma(k, n-1)\left(x_{i}^{\prime}\right) .
\end{aligned}
$$

Now computing $\sigma(j, n)-x_{i} \sigma(j-1, n)$ gives the result.

LEMMA 3. If $A_{i}$ are column vectors, then

$$
\begin{aligned}
\operatorname{det}\left(A_{1}-x A_{2}, A_{2}-x A_{3}, \cdots, A_{n}-x A_{n+1}\right) & \\
& =\operatorname{det}\left(\begin{array}{ccccc}
A_{1} & A_{2} & \cdots & A_{n} & A_{n+1} \\
x^{n} & x^{n-1} & \cdots & x & 1
\end{array}\right) .
\end{aligned}
$$

Proof. In $\operatorname{det}\left(A_{1}-x A_{2}, \cdots, A_{n}-x A_{n+1}\right)$ multiply the last column by $x$ and add to the previous column. In the resulting determinant multiply the second last column by $x$ and add to the third last column. Repeat this process to get

$$
\begin{aligned}
\operatorname{det}\left(A_{1}-x^{n} A_{n+1}, A_{2}-\right. & \left.x^{n-1} A_{n+1}, \cdots, A_{n}-x A_{n+1}\right) \\
& =\operatorname{det}\left(\begin{array}{ccc}
A_{1}-x^{n} A_{n+1}, \cdots, & A_{n}-x A_{n+1}, A_{n+1} \\
0 & , \cdots, & 0
\end{array}\right) \\
& =\operatorname{det}\left(\begin{array}{cccc}
A_{1} & A_{2} & A_{n} & A_{n+1} \\
x^{n} & x^{n-1} & x & 1
\end{array}\right) .
\end{aligned}
$$

We are now in a position to compute $g(0, n, m)$ by use of (2).

LEMMA 4. For $n \geqq 2, \quad m \geqq 1, \quad g(0, n, m)(x)=\operatorname{det}\left(b_{i j}\right)$ where $b_{i j}=$ $\sigma(m-1+i-j, n)$, and $B$ is an $(n-1) \times(n-1)$ matrix with constant diagonals.

Proof. By (7) we see that for $n=2$ we have $g(0,2, m)=\sigma(m-1, n)$ so we may proceed by induction. Note that if we define

$$
A(j, k)=(\sigma(j, k), \sigma(j+1, k), \cdots, \sigma(j+n-2, k))^{T}
$$

then the induction hypothesis is that

$$
g(0, n, m)=\operatorname{det}(A(m-1, n), A(m-2, n), \cdots, A(m-n+1, n)) .
$$


Thus from (2)

But using Lemmas 3 and 4,

$$
g(0, n+1, m)=\sum_{i=1}^{n+1} x_{i}^{m+n-1} \pi_{i} g(0, n, m)\left(x_{i}^{\prime}\right) .
$$

$$
\begin{aligned}
& g(0, n, m)\left(x_{i}^{\prime}\right)=\operatorname{det}( A(m-1, n+1)-x_{i} A(m-2, n+1), \\
& A(m-2, n+1)-x_{i} A(m-3, n+1), \\
&\left.\cdots, A(m-n+1, n+1)-x_{i} A(m-n, n+1)\right) \\
&=\operatorname{det}\left(\begin{array}{ccc}
A(m-1, n+1) & A(m-2, n+1) \\
x_{i}^{n-2} & x_{i}^{n-3} \\
\cdots & A(m-n+1, n+1) \\
\cdots & 1
\end{array}\right) .
\end{aligned}
$$

Putting this in the right-hand side of (8), and noting that only the last row depends on $x_{i}$,

$$
g(0, n+1, m)=\operatorname{det}\left(\begin{array}{cc}
A(m-1, n+1) & A(m-2, n+1) \\
\sum_{i=1}^{n+1} x_{i}^{m+2 n-3} \pi_{i} & \sum_{i=1}^{n+1} x_{i}^{m+2 n-4} \pi_{i} \\
A(m-n+1, n+1) \\
\sum_{i=1}^{n+1} x_{i}^{m+n-1} \pi_{i}
\end{array}\right)
$$

and using the Lemma 1 , the last row is

$$
(\sigma(m+n-3, n+1), \sigma(m+n-2, n+1), \cdots, \sigma(m-1, n+1))
$$

which agrees with the proposed formula for $g(0, n+1, m)$.

We are now able to write the general formula for all $g(\alpha, n, m)$. Define $h(\alpha, n, m)=g(\alpha, n, m+\alpha)$. Then the recursion (2) becomes

$$
h(\alpha, n, m)(x)=\sum_{i=1}^{n} x_{i}^{m+n-2} \pi_{i} h(\alpha, n-1, m)\left(x_{i}^{\prime}\right)
$$

and (7) becomes

$$
h(\alpha, \alpha+2, m)=g(\alpha, \alpha+2, m+\alpha)=\sigma(m-1, \alpha+2) .
$$

That is, $h(\alpha, n, m)$ satisfies the same recursion as $g(0, n, m)$ and has the same initial value, i.e. at $n=\alpha+2$, the only change required is in the number of variables. Thus a proof completely analogous to the proof of Lemma 4 will prove our main theorem. 
THEOREM. For $0 \leqq \alpha \leqq n-2, m=0,1,2, \cdots, g(\alpha, n, m)=\operatorname{det}\left(b_{i j}\right)$ where $b_{i j}=\sigma(m-1-\alpha+i-j, n)$ and $B$ is an $(n-\alpha-1) \times(n-\alpha-1)$ matrix.

An interesting corollary is that $g(\alpha+k, n, m+k)=g(\alpha, n, m)$ provided both are defined. Note that the case $m=n$ is the one of interest for our extremal problem posed earlier. One could attempt to write $b_{i j}$, and hence the functions $g$, in terms of the elementary symmetric functions, but this seems difficult to do. For example $g(n-2, n, n)=x_{1}+\cdots+x_{n}=\sigma_{1}$, which is very simple. However already for $n-\alpha=3$ the formula becomes much more complex. One can show that

$$
g(n-3, n, n)=\sum_{i \neq j} x_{i}^{2} x_{j}^{2}+\sum_{i \neq j \neq k} x_{i} x_{j} x_{k}^{2}+2 \sum_{i \neq j \neq k \neq l} x_{i} x_{j} x_{k} x_{l} .
$$

Comparing this with

$$
g(n-3, n, n)=\operatorname{det}\left(\begin{array}{cc}
\sum_{|k|=2} x_{1}^{k_{1}} \cdots x_{n}^{k_{n}} & \sum_{|k|=1} x_{1}^{k_{1}} \cdots x_{n}^{k_{n}} \\
\sum_{|k|=3} x_{1}^{k_{1}} \cdots x_{n}^{k_{n}} & \sum_{|k|=2} x_{1}^{k_{1}} \cdots x_{n}^{k_{n}}
\end{array}\right)
$$

one sees that there are many cancellations in the second form. In fact, the degree three terms present in the determinant form do not appear in the simplified form. If one goes back to the definition, one sees that as a polynomial in $x_{i}$, the degrees of $V(\alpha, n, m)$ and $V_{n}$ are $m+n-\alpha-2$ and $n-1$ respectively. Thus no term in $g(\alpha, n, m)$ has degree in $x_{i}$ greater than $m-\alpha-1$. This shows that there perhaps ought to be a better general representation for $g(\alpha, n, m)$.

\section{REFERENCE}

1. M. D. Hirschhorn, Amer. Math. Monthly 79 (1972), 518-519.

Department of Mathematics, Iowa State University, Ames, lowa 50010 